\title{
The Association of Urinary Sclerostin and Renal Magnesium Handling in Type 2 Diabetic Patients with Chronic Kidney Disease
}

\author{
Ching-Fang Wu ${ }^{a, b} \quad$ Hung-Hsiang Liou ${ }^{c, d} \quad$ Chin-Chi Kuo $^{e, f} \quad$ Ming-Hsien Tsai $^{9}$ \\ Min-Yu Chang ${ }^{a, d}$ Yi-Che Lee ${ }^{a, h}$ Tsun-Mei Lin ${ }^{i, j}$ Shih-Yuan Hung ${ }^{a, d}$ \\ aSchool of Medicine, College of Medicine, I-Shou University, Kaohsiung, Taiwan; 'bivision of Nephrology, \\ Department of Internal Medicine, E-Da Cancer Hospital, Kaohsiung, Taiwan; 'Division of Nephrology, Department \\ of Internal Medicine, Hsin-Jen Hospital, New Taipei City, Taiwan; dDivision of Nephrology, Department of Internal \\ Medicine, E-Da Hospital, Kaohsiung, Taiwan; 'Big Data Center, China Medical University Hospital, China Medical \\ University, Taichung, Taiwan; 'Division of Nephrology, Department of Internal Medicine, China Medical University \\ Hospital, Taichung, Taiwan; 9Division of Nephrology, Department of Internal Medicine, Shin-Kong Wu Ho-Su \\ Memorial Hospital, Taipei, Taiwan; hDivision of Nephrology, Department of Internal Medicine, E-Da Dachang \\ Hospital, Kaohsiung, Taiwan; 'Department of Medical Laboratory Science, I-Shou University, Kaohsiung, Taiwan; \\ 'Department of Medical Research, E-Da Hospital, Kaohsiung, Taiwan
}

\section{Keywords}

Sclerostin · Fractional excretion of calcium · Fractional excretion of magnesium - Type 2 diabetes mellitus · Chronic kidney disease

\begin{abstract}
Introduction: Sclerostin could enhance renal excretion of calcium (Ca) and phosphate ( $P$ ). The association between sclerostin and magnesium (Mg) has not yet discovered. In patients with type 2 diabetes mellitus (T2DM) or chronic kidney disease (CKD), higher serum sclerostin and altered renal excretion of $\mathrm{Ca}, \mathrm{P}$, and $\mathrm{Mg}$ were detected. Therefore, we tried to evaluate if there was any association between sclerostin and fractional excretion of $\mathrm{Ca}, \mathrm{P}$, and $\mathrm{Mg}$ ( $\mathrm{FeCa}$, FeP, and FeMg) in T2DM with CKD. Methods: In this prospective cohort study, 43 T2DM patients without CKD or with CKD stage 1-5 were enrolled. Values of parameters, including serum and urine sclerostin, were collected at baseline and 6 months later. For baseline data, the Mann-Whitney $U$ test, $X^{2}$ test, or
\end{abstract}

karger@karger.com www.karger.com/kbr

Karger"
(C) 2021 The Author(s)

Published by S. Karger AG, Basel

This is an Open Access article licensed under the Creative Commons Attribution-NonCommercial-4.0 International License (CC BY-NC) (http://www.karger.com/Services/OpenAccessLicense), applicable to the online version of the article only. Usage and distribution for commercial purposes requires written permission.
Spearman's correlation were used. For multivariate repeated measurement analysis, generalized estimating equation (GEE) model was utilized. Results: Patients with lower estimated glomerular filtration rate had higher serum sclerostin, FeP, FeMg, and lower FeCa. By correlation analysis, serum sclerostin was negatively associated with FeCa $(p=0.02)$ and positively associated with FeP $(p=0.002)$. The urine sclerostin to creatinine ratio (Uscl/Ucre) was positively correlated with FeP $(p=0.007)$ and FeMg $(p=0.005)$. After multivariate analyses by GEE model, serum sclerostin was still inversely associated with FeCa, while Uscl/Ucre was significantly associated with FeMg. On the other hand, FeP lost its associations with serum sclerostin or Uscl/Ucre. Conclusion: In our study population of T2DM patients with or without CKD, the inverse correlation between serum sclerostin and FeCa could not be explained by the calciuric effect of sclerostin. In addition, a

Ching-Fang Wu and Hung-Hsiang Liou contributed equally to this work.
Correspondence to:

Shih-Yuan Hung, ed100367@edah.org.tw 
newly discovered positive association between urinary sclerostin and FeMg indicated a possible role of urinary sclerostin in regulating renal $\mathrm{Mg}$ handling especially over distal convoluted tubules.

(c) 2021 The Author(s).

Published by S. Karger AG, Basel

\section{Introduction}

Sclerostin, encoded by SOST gene, is a secreted glycoprotein mainly produced by mature osteocytes. It is known to inhibit Wnt- $\beta$-catenin pathway, thus inhibiting osteoblast differentiation and subsequently bone formation [1]. Clinically, serum sclerostin level was higher in patients with type 2 diabetes mellitus (T2DM) or chronic kidney disease (CKD) [2]. In T2DM, higher serum sclerostin, possibly due to enhanced osteocyte production by hyperglycemia or inflammatory status [3-5], is recognized as a risk factor for osteoporosis [6]. While in CKD, higher serum sclerostin, possibly resulting from enhanced production instead of reduced renal clearance [7], has been shown to be associated with bone metabolism [7-10], arterial stiffness [11], vascular calcifications [12], cardiovascular events as well as mortality [13].

Various alterations of renal calcium $(\mathrm{Ca})$ and phosphate (P) handling are observed in T2DM and CKD. Hypercalciuria has been found in uncontrolled T2DM, which could be explained by osmotic diuresis, glucose ingestion, and decreased renal alpha-Klotho expression [14-16]. In addition, increased renal P loss has also been detected in T2DM, which may be the consequence of negative bone balance [16]. On the other hand, the decline in urinary Ca excretion during CKD progress could be partly explained by low $1 \alpha, 25$-dihydroxy vitamin $\mathrm{D}\left[1 \alpha, 25-(\mathrm{OH})_{2} \mathrm{D}\right][17]$ and high parathyroid hormone (PTH) levels [18]. In the opposite, the negative correlation between urinary $\mathrm{P}$ excretion and the glomerular filtration rate (GFR) $[17,19]$ is probably due to elevated fibroblast growth factor 23 (FGF23) and PTH in response to P retention [20]. Moreover, sclerostin was found not only to inhibit the synthesis of 1 a hydroxylase by cultured proximal tubular cells [21], but also to indirectly stimulate the secretion of FGF23 by osteoblasts [22]. Therefore, urinary Ca excretion was lower in sost ${ }^{-1-}$ mice than in wild type mice [21]. In addition, serum sclerostin was shown to be correlated with urinary $\mathrm{P}$ excretion in CKD population [7].

Renal handling of magnesium $(\mathrm{Mg})$ shares many similar regulatory mechanisms such as PTH, 1a,25-
$(\mathrm{OH})_{2} \mathrm{D}, \mathrm{Ca}$ sensing receptor, and claudin [23] as that of $\mathrm{Ca}$ and $\mathrm{P}$. In addition, enhanced renal $\mathrm{Mg}$ excretion was also found in CKD [24] and DM [25], but the underlying mechanisms were mainly based on assumptions. In CKD, it was believed to be the compensation for loss of renal function [24], while in DM, enhanced filtered load due to glomerular hyperfiltration or proteinuria or reduced renal reabsorption resulting from insulin deficiency or resistance might be the reason [25]. Since hypomagnesemia was linked to several chronic diabetic complications in T2DM patients [25], it is necessary to explore the underlying mechanism to further develop proper management against hypomagnesemia in this population.

Since sclerostin could promote renal $\mathrm{Ca}$ and $\mathrm{P}$ excretion at least by inhibiting production of $1 \alpha, 25-(\mathrm{OH})_{2} \mathrm{D}$ [21], it was interesting to investigate if sclerostin was linked to altered renal handling of $\mathrm{Ca}$ and $\mathrm{P}$ in T2DM or CKD. Moreover, sclerostin has not yet been shown to have any relationship with renal $\mathrm{Mg}$ handling. Since higher serum sclerostin and enhanced renal Mg excretion both existed in T2DM and CKD, it was of interest to know if sclerostin had any association with renal $\mathrm{Mg}$ handling in these populations. Accordingly, a prospective cohort study was designed to evaluate possible associations between sclerostin and urinary excretion of $\mathrm{Ca}, \mathrm{P}$, and $\mathrm{Mg}$ in T2DM patients within different stages of CKD.

\section{Patients and Methods}

\section{Study Population}

From September to December 2015, around 2,400 patients visiting Nephrology Outpatient Department in E-Da Hospital, Kaohsiung, had been screened. T2DM patients with age $\geq 20$ years, no new onset fracture, and no exposure to vitamin $\mathrm{D}$ and its analogues, diuretics, steroid, Ca-containing medications, or treatment for osteoporosis in recent 3 months were enrolled in this study. In opposite, patients with pregnancy, cancer, liver disease, renal replacement therapy including hemodialysis, peritoneal dialysis, and renal transplantation, or active infection were excluded. Finally, 43 patients were enrolled in this study. Written informed consent was obtained from these patients. The study was approved by the Institution Review Board of the hospital (number EMRP-104-045) and was in adherence with the Declaration of Helsinki.

\section{Demographic and Lab Data Collection}

For each patient, we recorded clinical parameters including age, sex, body mass index, the diagnosis of hypertension, and blood pressure. The medication for diabetes within 3 months before the acquisition of baseline data was also recorded. In addition, fasting blood and randomly spot urine samples were collected. Creatinine, albumin, $\mathrm{Ca}, \mathrm{P}, \mathrm{Mg}$, glycated hemoglobin (HbA1c), sclerostin, bone-specific alkaline phosphatase (BAP), collagen type 1 cross- 
linked C-telopeptide (CTX), intact PTH (iPTH), FGF23, 25-OHD, and soluble alpha-Klotho (sKlotho) were measured in serum or plasma. Estimated GFR (eGFR) was calculated using modification of diet in renal disease equation. On the other hand, urine total protein, creatinine, $\mathrm{Ca}, \mathrm{P}, \mathrm{Mg}$, and sclerostin were measured. Urine protein-creatinine ratio (UPCR), fractional excretion of $\mathrm{Ca}$, $\mathrm{P}$, and $\mathrm{Mg}(\mathrm{FeCa}, \mathrm{FeP}$, and $\mathrm{FeMg}$ ) were then calculated accordingly.

\section{Assays}

Serum creatinine, albumin, $\mathrm{Ca}, \mathrm{P}, \mathrm{Mg}$, and 25-OHD as well as plasma HbA1c and iPTH were measured at Department of Laboratory Medicine in E-Da Hospital. Serum creatinine was measured by the Jaffe method with Clinimate CRE reagent on TOSHIBAC16000 analyzer. With Abbott i2000 immunology analyzer, serum 25-OHD and plasma iPTH were assayed by chemiluminescent microparticle immunoassay using ARCHITECT 25-OH vitamin D reagent (Abbott, Germany) and ARCHITECT iPTH reagent (Abbott, Germany), respectively.

Serum and urine sclerostin were measured by enzymelinked immunosorbent assay (ELISA) (BI-20492; Biomedica, Austria) with inter- and intra-assay variation $\leq 10$ and $\leq 7 \%$, respectively. The detection limit was $3.2 \mathrm{pmol} / \mathrm{L}$. Serum BAP was measured by Ostase ${ }^{\circledR}$ BAP immunoenzymetric assay (AC$20 \mathrm{~F} 1$, IDS, UK) with both inter- and intra-assay variation $<7 \%$. The detection limit was $0.7 \mu \mathrm{g} / \mathrm{L}$. Serum CTX was measured by ELISA (SL0540Hu; SunLong Biotech, China) with inter- and intra-assay variation $<12$ and $<10 \%$, respectively. The detection limit was $12.5 \mathrm{pg} / \mathrm{mL}$. Serum FGF23 was assayed with a commercially available kit (CY-4,000; Kainos Lab, Japan) with inter- and intra-assay coefficients of variation $<5 \%$. The lower limit of detection was $3 \mathrm{pg} / \mathrm{mL}$. Serum sKlotho was assayed with ELISA $(27,988$; IBL, Japan) with within- and between-run variation $<5$ and $<8 \%$, respectively. The lower limit of detection was $6.15 \mathrm{pg} / \mathrm{mL}$.

\section{Statistical Analysis}

The normality of all parameters was analyzed by the ShapiroWilk normality test. Many parameters including serum sclerostin $(p=0.019)$, BAP $(p<0.001)$, FGF23 $(p=0.014)$, sKlotho $(p<0.001)$, plasma HbA1c $(p<0.001)$, iPTH $(p=0.001)$, UPCR $(p=0.009)$, and $\mathrm{FeCa}(p<0.001)$ were not normally distributed, and our sample size was relatively small. Therefore, nonparametric statistics were utilized in this study. Continuous variables were expressed as median (Q1, Q3) and categorical variables were expressed as frequency (percentage). To compare baseline parameters between low eGFR and high eGFR groups, the MannWhitney U test or $\chi^{2}$ test was adopted. In addition, Spearman's correlation was chosen for correlation analysis of baseline data. Finally, generalized estimating equation (GEE) was used for multivariate repeated measurement analysis. In GEE model, the covariance structure was set as first order autoregressive. Corrected quasi-likelihood under independence model criterion was applied for choosing the best sets of parameters. Lower corrected quasi-likelihood under independence model criterion indicated better fit. All statistical analyses were performed in SPSS version 19. Two-sided $p<0.05$ was considered as statistically significant.

\section{Results}

\section{Baseline Characteristics of Study Population}

Finally, 43 patients had their baseline data and 33 of them had their second data 6 months later. Their baseline characteristics are shown in Table 1 . The median age was 59 years and 31 patients $(72.1 \%)$ were male. Thirty-four $(79.1 \%)$ of them had hypertension. The median eGFR was $55.2 \mathrm{~mL} / \mathrm{min} / 1.73 \mathrm{~m}^{2}$ (13 without CKD, 4 in CKD stage 2, 21 in CKD stage 3, 2 in CKD with stage 4, and 3 in CKD stage 5), median UPCR was $321(74.9,1,207)$ $\mathrm{mg} / \mathrm{g}$, and median HbAlc was $6.9(6.60,8.20) \%$. The numbers of patients receiving different kinds of medication for T2DM were listed as below: 10 (23.3\%) for insulin, 18 (41.9\%) for metformin, 35 (81.4\%) for dipeptidyl peptidase 4 inhibitors, 31 (72.1\%) for sulfonylurea, 1 (2.3\%) for repaglinide, 15 (34.9\%) for acarbose, and 2 (4.7\%) for pioglitazone.

\section{Comparison between Patients with Lower or Higher eGFR}

These 43 patients were further divided into 2 groups according to their baseline eGFR: low eGFR $(<60 \mathrm{~mL} /$ $\left.\mathrm{min} / 1.73 \mathrm{~m}^{2}\right)$ or high eGFR $\left(\geq 60 \mathrm{~mL} / \mathrm{min} / 1.73 \mathrm{~m}^{2}\right)$. As shown in Table 1, patients with low eGFR were older in age $(p=0.005)$ and had higher serum sclerostin $(p=$ $0.009)$, FGF23 ( $p=0.003)$, UPCR $(p=0.001), \mathrm{FeP}(p=$ $0.004)$, and FeMg $(p<0.001)$ but lower FeCa $(p=0.03)$ compared to those with higher eGFR. On the other hand, there was no significant difference in systolic, diastolic, and mean blood pressure, serum $\mathrm{Ca}, \mathrm{P}, \mathrm{Mg}$, BAP, CTX, 25-OHD, and sKlotho, plasma iPTH and $\mathrm{HbAlc}$, and urine sclerostin to creatinine ratio (Uscl/ Ucre) between groups.

\section{Factors Associated with Serum and Urine Sclerostin}

By correlation analysis of baseline data (see online suppl. Table 1; see www.karger.com/doi/10.1159/000516844 for all online suppl. material), serum sclerostin was positively correlated with serum FGF23 $(r=0.507, p=$ $0.001)$, UPCR $(r=0.306, p=0.046)$, and FeP $(r=0.469$, $p=0.002)$, and negatively associated with eGFR $(r=$ $-0.517, p<0.001)$ and FeCa $(r=-0.355, p=0.046)$. Multivariate analysis by GEE model showed that after adjustment by eGFR, FGF23, UPCR, and FeP, serum sclerostin was still negatively associated with $\mathrm{FeCa}(r=$ $-5.350, p=0.037)$. In online suppl. Table 2 , baseline Uscl/Ucre was correlated positively with $\mathrm{FeP}(r=0.413$, $p=0.007)$ and FeMg $(r=0.424, p=0.005)$, and negatively with serum albumin $(r=-0.336, p=0.03)$. After 
Table 1. Baseline parameters of study population

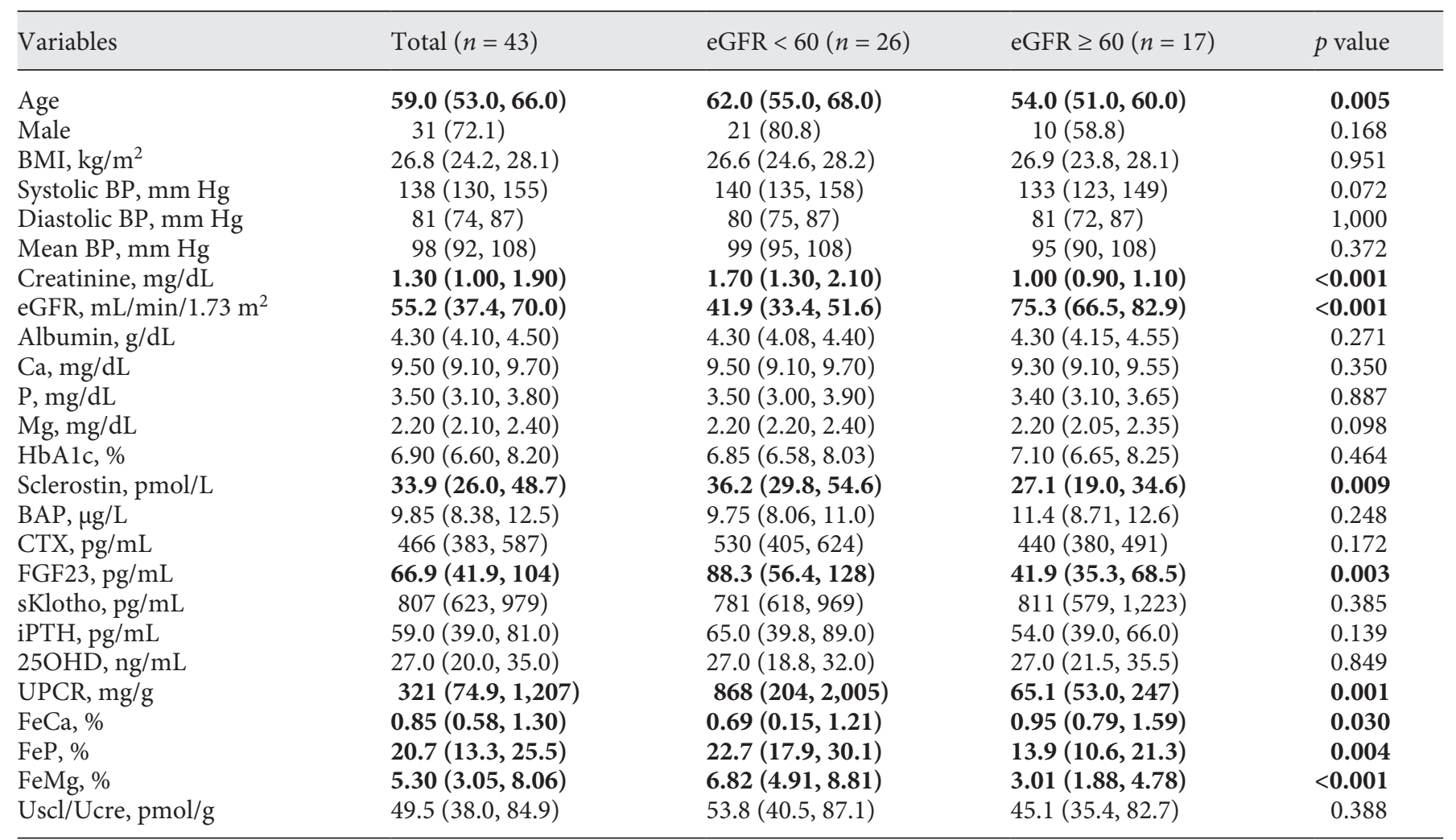

Male is expressed as the $n(\%)$, and other variables are expressed as the median (Q1, Q3). Bold indicates significant difference. BMI, body mass index; BAP, bone-specific alkaline phosphatase; BP, blood pressure; Ca, calcium; CTX, collagen type 1 cross-linked C-telopeptide; eGFR, estimated glomerular filtration rate; $\mathrm{FeCa}$, fractional excretion of calcium; FeMg, fractional excretion of magnesium; FeP, fractional excretion of phosphate; FGF23, fibroblast growth factor 23; HbA1c, glycated hemoglobin; iPTH, intact parathyroid hormone; Mg, magnesium; 25OHD, 25-hydroxy vitamin D; P, phosphate; sKlotho, soluble alpha-Klotho; UPCR, urine protein-creatinine ratio; Uscl/Ucre, urine sclerostin to creatinine ratio.

adjustment by serum albumin and FeP using GEE model, Uscl/Ucre was still associated positively with FeMg $(r=$ $4.102, p=0.005)$.

\section{The Association between Sclerostin and $\mathrm{FeCa}, \mathrm{FeP}$, and FeMg}

We further investigated the association between sclerostin and FeCa, FeP, or FeMg. As shown in Table 2, baseline serum sclerostin $(r=-0.355, p=0.020)$, not Uscl/ Ucre, was inversely correlated with FeCa by correlation analysis. The scatterplot of serum sclerostin and FeCa was shown in Figure 1a. After correcting for eGFR, FGF23, sKlotho, and UPCR (model 1) or eGFR, sKlotho, iPTH, and 25OHD (model 2) by GEE, FeCa was still inversely associated with serum sclerostin $(r=-0.008, p=0.034$ in model 1 and $r=-0.009, p=0.019$ in model 2). Besides, it is noteworthy that although iPTH was not correlated with $\mathrm{FeCa}$ in correlation analysis, it became negatively associated with FeCa in GEE model $2(r=-0.004, p=0.046)$.

In Table 3, baseline $\mathrm{FeP}$ was correlated with serum sclerostin $(r=0.469, p=0.002)$ and Uscl/Ucre $(r=0.413$, $p=0.007)$. After multivariate analysis by GEE model, both serum sclerostin and Uscl/Ucre lost their associations with $\mathrm{FeP}$. Instead, $\mathrm{FeP}$ was significantly associated with eGFR $(r=-0.273, p<0.001$ in model 1 and $r=$ $-0.237, p<0.001$ in model 2$)$ and iPTH $(r=0.053, p=$ 0.032 in model 2).

In Table 4, baseline FeMg was correlated with Uscl/Ucre $(r=0.424, p=0.005)$, not serum sclerostin. The scatterplot of Uscl/Ucre and FeMg is shown in Figure 1b. After correcting for age, eGFR, serum albumin, FGF23, UPCR, FeP (model 1) or eGFR, FGF23, and iPTH (model 2) by 
Table 2. Univariate correlation with FeCa and multivariate analyses by GEEs

\begin{tabular}{|c|c|c|c|c|c|c|c|c|}
\hline Variables & rho & $p$ value & $\begin{array}{l}\text { Coeffi. of GEE } \\
\text { model } 1\end{array}$ & $p$ value & QICC & $\begin{array}{l}\text { Coeffi. of GEE } \\
\text { model } 2\end{array}$ & $p$ value & QICC \\
\hline eGFR & 0.415 & 0.006 & 0.004 & 0.487 & & 0.003 & 0.579 & \\
\hline FGF23 & -0.504 & 0.001 & -0.001 & 0.472 & & & & \\
\hline sKlotho & 0.355 & 0.028 & 0.000 & 0.336 & 53.50 & 0.000 & 0.296 & 53.12 \\
\hline UPCR & -0.328 & 0.032 & $-2.777 \mathrm{E}-5$ & 0.691 & & & & \\
\hline $25 \mathrm{OHD}$ & -0.163 & 0.298 & & & & -0.015 & 0.088 & \\
\hline
\end{tabular}

Bold indicates significant difference. Coeffi., coefficient; eGFR, estimated glomerular filtration rate; FGF23, fibroblast growth factor 23; iPTH, intact parathyroid hormone; 25OHD, 25-hydroxy vitamin D; QICC, corrected quasi-likelihood under independence model criterion; sKlotho, soluble alpha-Klotho; UPCR, urine protein-creatinine ratio; FeCa, fractional excretion of calcium; GEEs, generalized estimating equations.

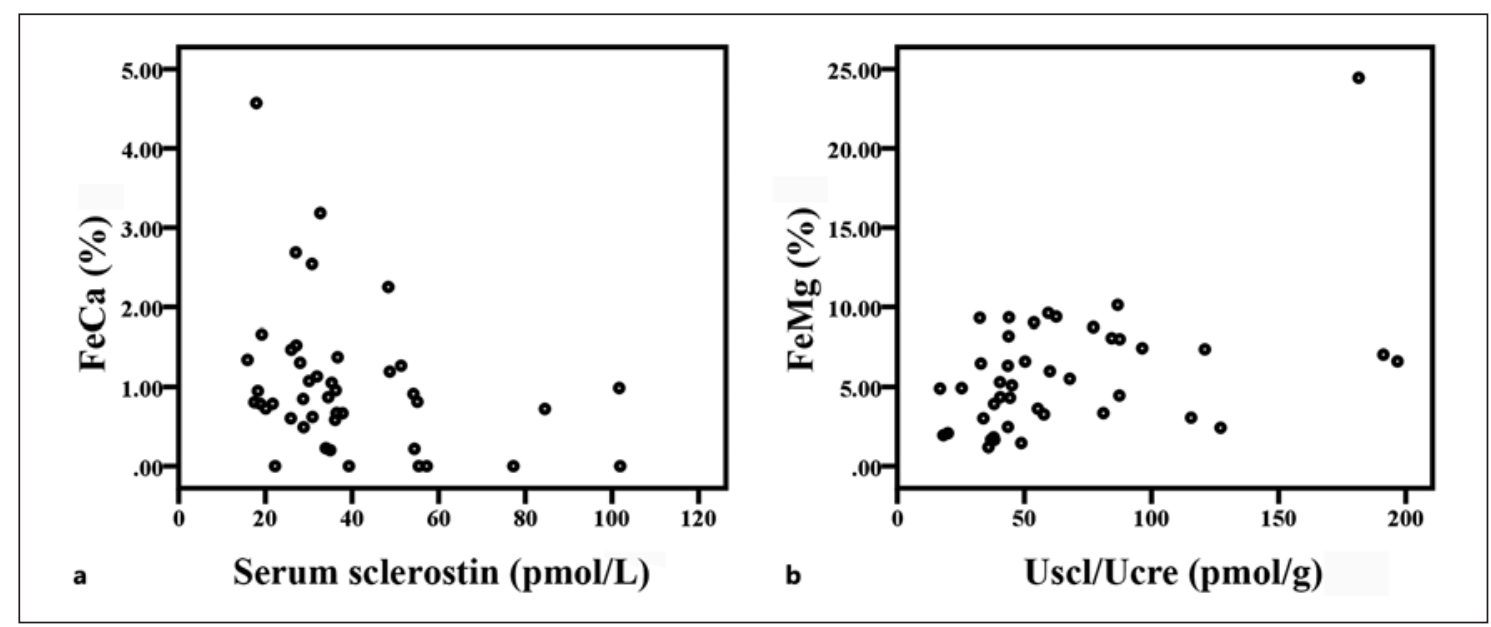

Fig. 1. The scatterplot of baseline serum sclerostin and FeCa (a), and baseline urinary sclerostin (represented as Uscl/Ucre) and FeMg (b). By Spearman's correlation, baseline serum sclerostin was inversely correlated with $\mathrm{FeCa}(r=-0.355, p=0.020)$, while baseline Uscl/Ucre was correlated with FeMg $(r=0.424, p=0.005)$. Uscl/Ucre, urine sclerostin to creatinine ratio; FeCa, fractional excretion of calcium; FeMg, fractional excretion of magnesium.

GEE, FeMg was still associated with Uscl/Ucre $(r=0.015$, $p=0.017$ in model 1 or $r=0.023, p=0.027$ in model 2 ). In addition, there was no significant association between $\mathrm{FeMg}$ and iPTH.

\section{Discussion}

In this study, we have successfully shown that in T2DM patients with or without CKD, sclerostin has impacts on renal excretion of $\mathrm{Ca}, \mathrm{P}$, and $\mathrm{Mg}$ in different aspects. Serum sclerostin had significant association with $\mathrm{FeCa}$; Uscl/Ucre, as a marker for urine sclerostin, was strongly associated with FeMg. In our study population, serum sclerostin has been shown to negatively correlate with $\mathrm{FeCa}$ even after adjustment by eGFR, sKlotho, $\mathrm{iPTH}$, and 25OHD. It indicates that sclerostin is not responsible for hypercalciuria seen in T2DM. Indeed, hypercalciuric effect in T2DM could be due to the osmotic effect [26] or decreased renal alpha-Klotho expression [15]. In addition, sclerostin was considered to increase renal Ca excretion by downregulation of vitamin $\mathrm{D}$ activity [27]. Therefore, the inverse relationship between serum sclerostin and $\mathrm{FeCa}$ in our T2DM patients with or without CKD may reflect a possible new negative feedback of $\mathrm{FeCa}$ on sclerostin, which has not yet been 
Table 3. Univariate correlation with FeP and multivariate analyses by GEEs

\begin{tabular}{|c|c|c|c|c|c|c|c|c|}
\hline Variables & rho & $p$ value & $\begin{array}{l}\text { Coeffi. of GEE } \\
\text { model } 1\end{array}$ & $p$ value & QICC & $\begin{array}{l}\text { Coeffi. of GEE } \\
\text { model } 2\end{array}$ & $p$ value & QICC \\
\hline eGFR & -0.626 & $<0.001$ & -0.273 & $<0.001$ & \multirow{6}{*}{3,399} & -0.237 & $<0.001$ & \multirow{6}{*}{3,102} \\
\hline Albumin & -0.352 & 0.021 & -0.915 & 0.839 & & & & \\
\hline Sclerostin & 0.469 & 0.002 & 0.000 & 0.992 & & 0.011 & 0.746 & \\
\hline FGF23 & 0.366 & 0.016 & 0.010 & 0.446 & & 0.014 & 0.318 & \\
\hline Uscl/Ucre & 0.413 & 0.007 & 0.022 & 0.314 & & 0.034 & 0.090 & \\
\hline iPTH & 0.267 & 0.083 & & & & 0.053 & 0.032 & \\
\hline
\end{tabular}

Bold indicates significant difference. Coeffi., coefficient; eGFR, estimated glomerular filtration rate; FeMg, fractional excretion of magnesium; FGF23, fibroblast growth factor 23; iPTH, intact parathyroid hormone; QICC, corrected quasi-likelihood under independence model criterion; UPCR, urine protein-creatinine ratio; Uscl/Ucre, urine sclerostin to creatinine ratio; GEEs, generalized estimating equations; FeP, fractional excretion of phosphate.

Table 4. Univariate correlation with FeMg and multivariate analyses by GEEs

\begin{tabular}{|c|c|c|c|c|c|c|c|c|}
\hline Variables & rho & $p$ value & $\begin{array}{l}\text { Coeffi. of GEE } \\
\text { model } 1\end{array}$ & $p$ value & QICC & $\begin{array}{l}\text { Coeffi. of GEE } \\
\text { model } 2\end{array}$ & $p$ value & QICC \\
\hline Age & 0.350 & 0.021 & 0.014 & 0.779 & \multirow{7}{*}{421} & & & \multirow{7}{*}{506} \\
\hline eGFR & -0.648 & $<0.001$ & -0.093 & 0.001 & & -0.111 & $<0.001$ & \\
\hline Albumin & -0.343 & 0.025 & -2.819 & 0.079 & & & & \\
\hline FGF23 & 0.359 & 0.018 & -0.003 & 0.572 & & 0.000 & 0.933 & \\
\hline UPCR & 0.427 & 0.004 & 0.001 & 0.214 & & & & \\
\hline Uscl/Ucre & 0.424 & 0.005 & 0.015 & 0.017 & & 0.023 & 0.027 & \\
\hline iPTH & 0.234 & 0.131 & & & & 0.004 & 0.676 & \\
\hline
\end{tabular}

Bold indicates significant difference. Coeffi., coefficient; eGFR, estimated glomerular filtration rate; FeP, fractional excretion of phosphate; FeMg, fractional excretion of magnesium; FGF23, fibroblast growth factor 23; iPTH, intact parathyroid hormone; QICC, corrected quasi-likelihood under independence model criterion; UPCR, urine protein-creatinine ratio; Uscl/Ucre, urine sclerostin to creatinine ratio; GEEs, generalized estimating equations.

described before. Further studies are needed to confirm this hypothesis.

Compared with serum sclerostin, urinary sclerostin was seldom investigated before. In CKD population, urinary sclerostin, Uscl/Ucre, and fractional excretion of sclerostin, remained relatively low in CKD stage 1-3 but dramatically increased with wider variability when eGFR $<30 \mathrm{~mL} / \mathrm{min} / 1.73 \mathrm{~m}^{2}$ [7]. Based on the detection of sclerostin in human proximal tubular cells [7] and its relatively low molecular mass ( $28 \mathrm{kDa})$, sclerostin was estimated to be filtered through glomerular basement membrane and appear in urine if its filtered amount exceeds the reabsorptive capacity of tubular cells [7]. On the other hand, renal sclerostin expression was also demonstrated in the human kidney tissue [27], but the exact lo- cation for renal sclerostin expression was not identified. Therefore, the appearance of sclerostin in urine may be due to its filtered amount exceeding renal tubular reabsorptive capacity or increased renal production. In this study, Uscl/Ucre was not correlated with eGFR, which may be due to an uneven distribution of CKD stages in our patients (only 5 in CKD stage 4-5).

It is a novel finding to show the significant association of Uscl/Ucre and FeMg in our study population. In the kidney, about $96 \%$ of filtered $\mathrm{Mg}$ is reabsorbed by tubules (40-70\% in thick ascending limb [TAL] and $5-10 \%$ in distal convoluted tubule [DCT]). While Mg reabsorption in TAL shares the same paracellular pathway with Ca reabsorption, $\mathrm{Mg}$ reabsorption in DCT, acting through active transcellular pathway, fine-tunes the final renal $\mathrm{Mg}$ 


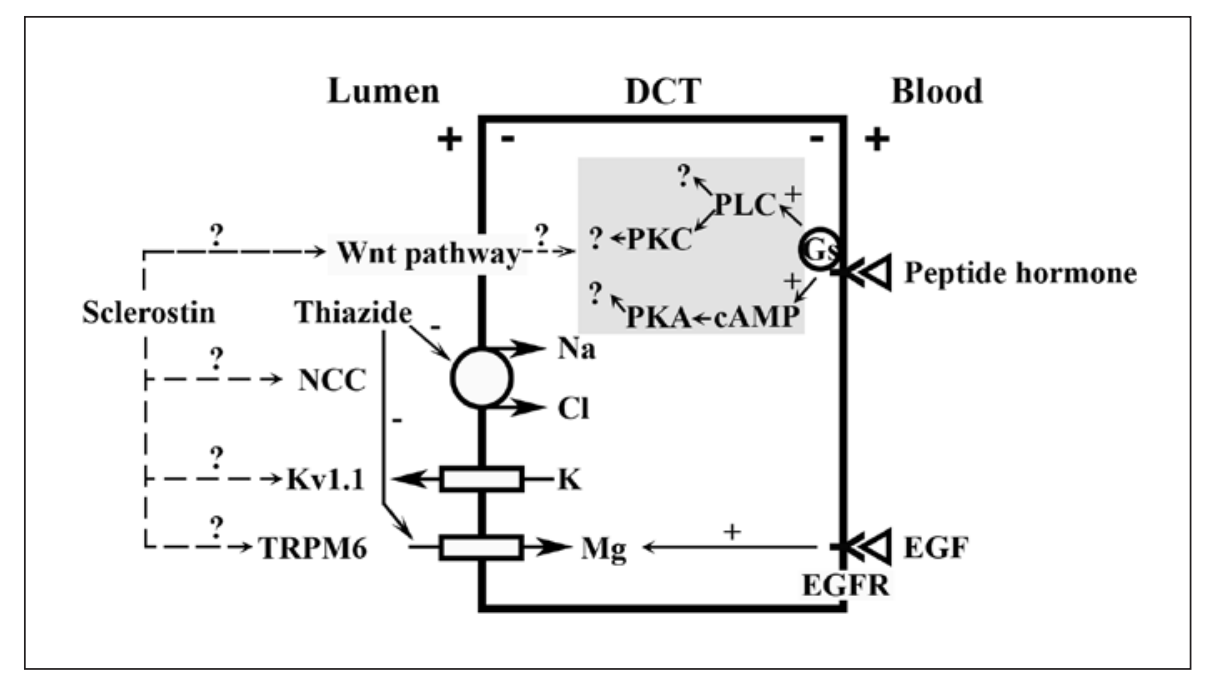

Fig. 2. A simplified diagram illustrating the possible role of sclerostin in renal Mg handling in DCT especially in T2DM with or without CKD. On the apical membrane of DCT, the voltage-gated potassium channel Kv1.1 facilitates Mg entry into cells via melastatinrelated TRPM6 by creating polarization of apical membrane. In addition, inhibition of NCC activity, such as thiazides, leads to renal $\mathrm{Mg}$ wasting possibly by downregulation of TRPM6. On the basolateral side of DCT, binding of EGF to its receptor EGFR can increase the activation and surface expression of TRPM6. Besides, peptide hormones such as PTH, calcitonin, glucagon, and even vasopressin can regulate $\mathrm{Mg}$ reabsorption partly by stimulation of cAMP release and activation of PKA as well as activation of PLC

excretion. On the apical membrane of DCT, the voltagegated potassium channel Kv1.1 creates polarization of apical membrane, driving $\mathrm{Mg}$ to enter the cell via the $\mathrm{Mg}$ channel, melastatin-related transient receptor potential cation channel 6 (TRPM6). The epidermal growth factor can bind to the epidermal growth factor receptor on the basolateral membrane of DCT and finally increases the activation and surface expression of TRPM6 $[23,28]$. In addition, inhibition of apical sodium-chloride cotransporter by thiazides was also shown to enhance Mg excretion by downregulation of TRPM6 [29]. Moreover, PTH, calcitonin, glucagon, and even vasopressin were all implicated to regulate $\mathrm{Mg}$ reabsorption at DCT at least partly by stimulation of cyclic adenosine monophosphate release and activation of protein kinase $\mathrm{A}$ as well as activation of phospholipase $\mathrm{C}$ and then protein kinase $\mathrm{C}$ [30]. Importantly, no data have linked sclerostin to renal $\mathrm{Mg}$ handling yet. In this study, Uscl/Ucre was found to be significantly associated with $\mathrm{FeMg}$, but not FeCa. Therefore, we hypothesize that sclerostin in urine may regulate $\mathrm{Mg}$ reabsorption in DCT instead of TAL. In addition, Uscl/Ucre, instead of serum sclerostin, was significantly and then PKC. In patients with T2DM with or without CKD, sclerostin may reach the urinary luminal side of DCT and then either directly influences apical TRPM6, Kv1.1, or even NCC activity or indirectly affect $\mathrm{Mg}$ reabsorption via possible interaction between intracellular Wnt signaling transduction pathway and PKA, PLC, or PKC. Mg, magnesium; T2DM, type 2 diabetes mellitus; CKD, chronic kidney disease; PTH, parathyroid hormone; DCTs, distal convoluted tubules; NCC, sodium-chloride cotransporter; TRPM6, transient receptor potential cation channel 6; EGF, epidermal growth factor; EGFR, epidermal growth factor receptor; cAMP, cyclic adenosine monophosphate; PKA, protein kinase A; PLC, phospholipase C; PKC, protein kinase C.

associated with FeMg. It is plausible that compared with its systemic effect, the local effect of sclerostin, most likely acting via urinary luminal side, plays a more important role in mediating $\mathrm{Mg}$ reabsorption in DCT. On DCT, sclerostin may either directly influence apical TRPM6, Kv1.1, or even sodium-chloride cotransporter activity or indirectly affect $\mathrm{Mg}$ reabsorption via possible interaction between intracellular Wnt signaling transduction pathway and protein kinase $\mathrm{A}$, phospholipase $\mathrm{C}$, or protein kinase $\mathrm{C}$. The possible role of sclerostin in renal Mg handling in DCT was summarized in Figure 2. However, currently no evidence has demonstrated the molecular mechanism by which sclerostin acts on renal tubules.

This study has some limitations. First, our study population was restricted to T2DM with uneven distribution of CKD stages. In addition, the number of our study population was relatively small. Nevertheless, repeated measurement analysis was utilized in this study to overcome the limitation of small sample size. Fortunately, we still discovered the interesting association between urinary sclerostin and FeMg. But further studies are needed to scrutinize this association in other populations. Besides, 
the mechanisms for sclerostin in regulating Mg reabsorption in DCT are also necessary to be explored.

In conclusion, this is the first study to show that in T2DM patients without or with CKD, serum sclerostin was inversely associated with renal Ca excretion. On the other hand, urinary sclerostin was positively associated with renal $\mathrm{Mg}$ excretion. Our findings seem to extend not only the role of sclerostin from serum to urine, but also its impacts from bone homeostasis to renal handling of minerals.

\section{Acknowledgments}

The authors would like to thank technical assistance of ChiuYa Liao and Ching-Cheng Wang.

\section{Statement of Ethics}

Written informed consent was obtained from patients enrolled. The study was approved by the Institution Review Board of E-Da Hospital (number EMRP-104-045) and was in adherence with the Declaration of Helsinki.

\section{Conflict of Interest Statement}

The authors have no conflicts of interest to declare.

\section{Funding Sources}

This study was supported by E-Da Hospital, E-Da Cancer Hospital, and I-Shou University (project EDAHP105008, EDPJ106052, EDCHP107006, and ISU-106-IUC-10).

\section{Author Contributions}

Concept and design of study: C.F. Wu, H.H. Liou, and S.Y. Hung. Acquisition of data: C.F. Wu, M.Y. Chang, Y.C. Lee, S.Y. Hung, and T.M. Lin. Statistical analysis of data: C.C. Kuo and M.H. Tsai. Drafting manuscript: C.F. Wu. Revising manuscript critically: H.H. Liou.

\section{References}

1 Compton JT, Lee FY. A review of osteocyte function and the emerging importance of sclerostin. J Bone Joint Surg Am. 2014 Oct 1; 96(19):1659-68.

2 Honasoge M, Rao AD, Rao SD. Sclerostin: recent advances and clinical implications. Curr Opin Endocrinol Diabetes Obes. 2014 Dec;21(6):437-46.

3 Baek K, Hwang HR, Park HJ, Kwon A, Qadir AS, Ko SH, et al. TNF-a upregulates sclerostin expression in obese mice fed a high-fat diet. J Cell Physiol. 2014 May;229(5):64050.

4 Kang J, Boonanantanasarn K, Baek K, Woo KM, Ryoo HM, Baek JH, et al. Hyperglycemia increases the expression levels of sclerostin in a reactive oxygen species- and tumor necrosis factor-alpha-dependent manner. J Periodontal Implant Sci. 2015 Jun;45(3):101-10.

5 Tanaka K, Yamaguchi T, Kanazawa I, Sugimoto T. Effects of high glucose and advanced glycation end products on the expressions of sclerostin and RANKL as well as apoptosis in osteocyte-like MLO-Y4-A2 cells. Biochem Biophys Res Commun. 2015 May 29;461(2): 193-9.

6 Wang N, Xue P, Wu X, Ma J, Wang Y, Li Y. Role of sclerostin and dkk1 in bone remodeling in type 2 diabetic patients. Endocr Res. 2018;43(1):29-38.
7 Cejka D, Marculescu R, Kozakowski N, Plischke M, Reiter T, Gessl A, et al. Renal elimination of sclerostin increases with declining kidney function. J Clin Endocrinol Metab. 2014 Jan; 99(1):248-55.

8 Cejka D, Herberth J, Branscum AJ, Fardo DW, Monier-Faugere MC, Diarra D, et al. Sclerostin and Dickkopf- 1 in renal osteodystrophy. Clin J Am Soc Nephrol. 2011 Apr; 6(4):877-82.

9 Ishimura E, Okuno S, Ichii M, Norimine K, Yamakawa T, Shoji S, et al. Relationship between serum sclerostin, bone metabolism markers, and bone mineral density in maintenance hemodialysis patients. J Clin Endocrinol Metab. 2014 Nov;99(11):4315-20.

10 Kuo TH, Lin WH, Chao JY, Wu AB, Tseng CC, Chang YT, et al. Serum sclerostin levels are positively related to bone mineral density in peritoneal dialysis patients: a crosssectional study. BMC Nephrol. 2019 Jul 17; 20(1):266.

11 Hsu BG, Liou HH, Lee CJ, Chen YC, Ho GJ, Lee MC. Serum sclerostin as an independent marker of peripheral arterial stiffness in renal transplantation recipients: a cross-sectional study. Medicine. 2016 Apr;95(15): e3300.

12 Yang CY, Chang ZF, Chau YP, Chen A, Yang $\mathrm{WC}$, Yang $\mathrm{AH}$, et al. Circulating Wnt $/ \beta$ catenin signalling inhibitors and uraemic vascular calcifications. Nephrol Dial Transplant. 2015 Aug;30(8):1356-63.
13 Evenepoel P, D'Haese P, Brandenburg V. Sclerostin and DKK1: new players in renal bone and vascular disease. Kidney Int. 2015 Aug;88(2):235-40.

14 Lee CT, Lien YH, Lai LW, Chen JB, Lin CR, Chen HC. Increased renal calcium and magnesium transporter abundance in streptozotocin-induced diabetes mellitus. Kidney Int. 2006 May;69(10):1786-91.

15 Asai O, Nakatani K, Tanaka T, Sakan H, Imura A, Yoshimoto S, et al. Decreased renal a-Klotho expression in early diabetic nephropathy in humans and mice and its possible role in urinary calcium excretion. Kidney Int. 2012 Mar;81(6):539-47.

16 Chen H, Li X, Yue R, Ren X, Zhang X, Ni A. The effects of diabetes mellitus and diabetic nephropathy on bone and mineral metabolism in T2DM patients. Diabetes Res Clin Pract. 2013 May;100(2):272-6.

17 Viaene L, Meijers BK, Vanrenterghem Y, Evenepoel P. Evidence in favor of a severely impaired net intestinal calcium absorption in patients with (early-stage) chronic kidney disease. Am J Nephrol. 2012;35(5):434-41.

18 Cozzolino M, Ciceri P, Volpi EM, Olivi L, Messa PG. Pathophysiology of calcium and phosphate metabolism impairment in chronic kidney disease. Blood Purif. 2009;27(4):33844.
Sclerostin and Renal Magnesium

Excretion in Diabetes with CKD
Kidney Blood Press Res 2021;46:514-522 DOI: $10.1159 / 000516844$ 
19 Tabibzadeh N, Mentaverri R, Daroux M, Mesbah R, Delpierre A, Paul JG, et al. Differential determinants of tubular phosphate reabsorption: insights on renal excretion of phosphates in kidney disease. Am J Nephrol. 2018;47(5):300-3.

20 Molony DA, Stephens BW. Derangements in phosphate metabolism in chronic kidney diseases/endstage renal disease: therapeutic considerations. Adv Chronic Kidney Dis. 2011 Mar;18(2):120-31.

21 Ryan ZC, Ketha H, McNulty MS, McGee-Lawrence M, Craig TA, Grande JP, et al. Sclerostin alters serum vitamin D metabolite and fibroblast growth factor 23 concentrations and the urinary excretion of calcium. Proc Natl Acad Sci U S A. 2013 Apr 9;110(15):6199-204.

22 Rowe PS. Regulation of bone-renal mineral and energy metabolism: the PHEX, FGF23, DMP1, MEPE ASARM pathway. Crit Rev Eukaryot Gene Expr. 2012;22(1):61-86.
23 Blaine J, Chonchol M, Levi M. Renal control of calcium, phosphate, and magnesium homeostasis. Clin J Am Soc Nephrol. 2015 Jul 7; 10(7):1257-72.

24 Cunningham J, Rodríguez M, Messa P. Magnesium in chronic kidney disease Stages 3 and 4 and in dialysis patients. Clin Kidney J. 2012 Feb;5(Suppl 1):i39-51.

25 Pham PC, Pham PM, Pham SV, Miller JM, Pham PT. Hypomagnesemia in patients with type 2 diabetes. Clin J Am Soc Nephrol. 2007 Mar;2(2):366-73.

26 Anwana AB, Garland HO. Renal calcium and magnesium handling in experimental diabetes mellitus in the rat. Acta Endocrinol. 1990 Apr;122(4):479-86.
27 Kumar R, Vallon V. Reduced renal calcium excretion in the absence of sclerostin expression: evidence for a novel calcium-regulating bone kidney axis. J Am Soc Nephrol. 2014 Oct; 25(10):2159-68.

28 Curry JN, Yu ASL. Magnesium handling in the kidney. Adv Chronic Kidney Dis. 2018 May;25(3):236-43.

29 Nijenhuis T, Vallon V, van der Kemp AW, Loffing J, Hoenderop JG, Bindels RJ. Enhanced passive $\mathrm{Ca} 2+$ reabsorption and reduced $\mathrm{Mg} 2+$ channel abundance explains thiazide-induced hypocalciuria and hypomagnesemia. J Clin Invest. 2005 Jun;115(6):1651-8.

30 Dai LJ, Ritchie G, Kerstan D, Kang HS, Cole DE, Quamme GA. Magnesium transport in the renal distal convoluted tubule. Physiol Rev. 2001 Jan;81(1):51-84. 